\title{
Guest Editorial Electromagnetic Crystal Structures, Design, Synthesis, and Applications
}

A NEW paradigm has emerged in which the band structure concepts of solid-state physics are applied to electromagnetics. This has led to a profusion of scientific creativity as new forms of electromagnetic crystal structures are invented for radio and microwaves as well as for optical wavelengths. These new structures are inspired by the three-dimensional (3-D) geometry of both natural crystals and those artificial crystals that can arise only in the human imagination.

These artificial electromagnetic crystals (also known as photonic crystals) are impacting the diverse domains of electromagnetics, extending from radio waves to optical wavelengths. They are bringing scientists together under a common umbrella in the fields of classical electromagnetics, solidstate band theory, semiconductor device physics, quantum optics, nanostructures, and materials science. The number of papers in this field have been growing at roughly $70 \%$ per year since the early 1990's, and there are currently hundreds of papers per year being published on photonic crystals.

There have already been a number of books [1], special journal issues [2], [3], and conference proceedings [4], [5] on this topic, originating from the very first one [2], organized by Bowden, Dowling, and Everitt, in Park City, UT, January 1992, extending to the "Workshop on Electromagnetic Crystal Structures," Laguna Beach, CA, 4-6 January 1999, the source of most of the papers in this issue. This series will now likely extend through the next international conference called, "Photonic and Electromagnetic Crystal Structures" to be held in Sendai, Japan, 8-10 March 2000.

Owing to the large number of papers that were submitted on the occasion of the January 1999 "Workshop on Electromagnetic Crystal Structures," the articles had to be divided among two journals, producing a pair of special issues. The papers that relate to radio and microwaves are in the companion November 1999 issue of the IEEE TRANSACTIONS ON MiCROWAVE THEORY AND TECHNIQUES, while the optically related papers are in this November 1999 issue of the JOURNAL OF LightwaVE TECHNOLOGY.

Photonic bandgaps (PBG's) originated from a pair of papers that were published almost simultaneously in 1987. One by Yablonovitch [6] introduced the forbidden gap for controlling spontaneous and stimulated emission of light. The second by John [7] introduced gaps to induce Anderson localization of light waves. In exploring analogies with low-energy electron diffraction (LEED), Ohtaka [8] in 1979 actually first used the

Publisher Item Identifier S 0733-8724(99)08930-6. phrase "photon band structure," but he narrowly missed the problematic concept of a photonic bandgap. Indeed, there was no assurance in 1987 that any photonic bandgap could ever be produced experimentally with available refractive indexes. The search for a 3-D photonic bandgap entailed numerous blind alleys and false starts, culminating in 1990 with the remarkable discovery [9] that diamond crystal geometry was favored by nature. This led to the first experimental demonstration [10] of a 3-D photonic bandgap.

Today, many more types of electromagnetic crystal structures and designs are being investigated in various dimensions and made of various materials, including metals. Even acoustic band structure is under study. Self-assembly and microfabrication have emerged as the main routes toward 3D optical structures. In addition to the 3-D photonic crystals, an important role will be played by $2-\mathrm{D}$, thin-film, photonic crystals. These are a good compromise between the total electromagnetic confinement of a 3-D structure versus the ease of fabrication of a 2-D patterned thin film. They appear to be capable of $Q$-factor up to 10000 in spite of being open structures, more than enough for lasing. In a milestone for the field, Painter et al. [11] report here lasing in a nanocavity, the smallest laser ever made. Thus, 2-D thin-film photonic crystals may form the underlying basis for a future technology of photonic integrated circuits.

While these dielectric structures have been very novel and exciting, a new element of richness has been introduced into electromagnetic/photonic crystal design recently with the incorporation of metallic components within the unit cells, i.e., metallo-dielectric photonic crystals. The physics of metallo-dielectric electromagnetic crystals is still being worked out, but it is clear that they follow their own set of rules, very different from the existing rules for dielectric structures. Thus, metallo-dielectric photonic crystals, both at radio frequencies, and optical frequencies, behave quite distinctly from the dielectric crystal structures that were previously explored. At the same time, they present entirely new, and very significant, technological and scientific opportunities.

At radio and microwave frequencies, the constraint linking spatial period and electromagnetic frequency has been undone. By capacitive loading between the metallic elements, the electromagnetic "valence band" frequency can be pushed down, thereby, electromagnetic crystal structures become valuable at radio frequencies. They become smaller and much more lightweight. Attention should be directed toward the paper by 
Sievenpiper et al. [12] in which a metal surface is converted to an insulator having a forbidden bandgap. It is expected that those types of high-impedance ground planes will be quite useful for antennas and for other applications in radio electromagnetics.

In the radio domain, PBG structures should not be confused with effective media, or frequency selective surfaces. The key point is that the electromagnetic wave vector component along the direction of periodicity is comparable to the reciprocal periodicity. In that case, the behavior of the wave-vector dispersion near a Brillouin Zone boundary becomes important. Band structure becomes imperative, and an effective dielectric constant or surface impedance provides an incomplete description.

At optical frequencies, the metallo-dielectric structures become strongly influenced by plasmon resonance, arising from electron inertia in the metallic constituent. Plasma wave polaritons (plasmons) can have frequencies in the optical regime combined with wavelengths much shorter than the corresponding vacuum electromagnetic wavelength. The resulting very large wave-vectors correspond to those of X rays! This permits the design of very tiny electromagnetic cavities, much smaller than anything currently being considered. The concentration of zero-point electromagnetic energy in such tiny plasmon cavities can produce a giant Purcell effect, enhanced by many orders of magnitude, leading to spontaneous emission that is much stronger than even stimulated emission. There is a link between the Purcell effect for spontaneous emission, and the surface-enhanced Raman effect for spontaneous Raman scattering, which is likewise enhanced by many orders of magnitude. There are but a few papers on this topic in this special JOURNAL OF LightwAVE TECHNOLOGY issue, but the plasmon band structure field is in its infancy, and we can expect to learn much more in the future.

The full, practical impact of PBG structures is yet to be seen, but we may observe the first commercial and military applications in the realm of handheld wireless communications and global positioning system (GPS) antennas using the highimpedance ground plane structures. The optical applications are more long range, but we can expect an impact on white pigments that are ubiquitous in our surroundings, on optical signal processing, and on light-emitting diodes (LED's), for both commercial and military use.

Given the evident and tangible progress in the field, it is clear that it will soon outgrow special topics issues. The spirit of comradeship and adventure among the researchers will remain as we continue to explore the realm of photonic crystal structures. We do not know what extra surprises nature will have in store for us.

\author{
AXEL SCHERER, Guest Editor \\ California Institute of Technology \\ Pasadena, CA 91125 USA
}

THEOdORE Doll, Guest Editor

California Institute of Technology

Pasadena, CA 91125 USA
Eli Yablonovitch, Guest Editor
University of California at
Los Angeles
Los Angeles, CA 90022 USA

HENRY O. EVERITT, Guest Editor

U.S. Army Research Office

Triangle Park, NC 27709 USA

John Aiden HigGins, Guest Editor

Rockwell Science Center

Thousand Oaks, CA 91360 USA

\section{REFERENCES}

[1] J. D. Joannopoulos, R. D. Meade, and J. N. Winn, Photonic Crystals. Princeton NJ: Princeton University Press 1995.

[2] C. M. Bowden, J. P. Dowling, and H. O. Everitt, "Development and applications of materials exhibiting photonic band gaps," J. Opt. Soc. Amer., vol. B10, p. 280, Feb. 1993.

[3] G. Kurizki and J. W. Haus, "Special issue on photonic band structures," J. Mod. Opt., vol. 41, p. 171, Feb. 1994.

[4] C. M. Soukoulis, Ed., "Photonic band gap materials," in Proc. NATO Advanced Study Inst. Photonic Band Gap Materials, Elounda, Crete, Greece, June 18-30, 1995; see also, Series E: Applied Sciences. Dordrecht, Germany: Kluwer Academic, 1996, vol. 315.

[5] E. Burstein and C. Weisbuch, "Confined electrons and photons: New physics and applications," in Proc. NATO Advanced Study Inst. Confined Electrons and Photons: New Physics and Applications, Italy, July 13-26, 1993; see also in Series B: Physics. New York: Plenum, 1995, vol. 340.

[6] E. Yablonovitch, "Inhibited spontaneous emission in solid-state physics and electronics," Phys. Rev. Lett., vol. 58, p. 2059, 1987.

[7] S. John, "Strong localization of photons in certain disordered dielectric superlattices," Phys. Rev. Lett., vol. 58, p. 2486, 1987.

[8] K. Ohtaka, "Energy band of photons and low-energy photon diffraction," Phys. Rev., vol. B19, p. 5057, 1979.

[9] K. M. Ho, C. T. Chan, and C. M. Soukoulis, "Existence of a photonic gap in periodic dielectric structures," Phys. Rev. Lett., vol. 65, p. 3152, 1990.

[10] E. Yablonovitch, T. J. Gmitter, and K. M. Leung, "Photonic band structure: The face-centered-cubic case employing nonspherical atoms," Phys. Rev. Lett., vol. 67, p. 2295, 1991

[11] O. J. Painter, A. Hussain, A. Scherer, J. D. O'Brien, I. Kim, and P. D. Dapkus, "Room temperature photonic crystal defect lasers at near infrared wavelengths in InGaAsP," this issue, pp. 2082-2088.

[12] D. Sievenpiper, L. Zhang, R. F. J. Broas, N. G. Alexópoulos, and E. Yablononvitch, "High-impedance electromagnetic surfaces with a forbidden frequency band," IEEE Trans. Microwave Theory Tech., vol. 47, pp. 2059-2074, Nov. 1999.

Axel Scherer, photograph and biography not available at the time of publication.

Theodore Doll, photograph and biography not available at the time of publication. 


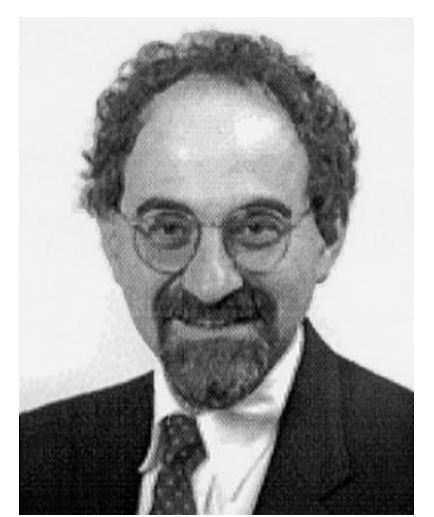

Eli Yablonovitch (M'75-SM'90-F'92) received the Ph.D. degree in applied physics from Harvard University, Cambridge, MA, in 1972.

He was with Bell Telephone Laboratories for two years and then became a Professor of applied physics at Harvard University. In 1979, he joined the Exxon Corporation to perform research on photovoltaic solar energy. In 1984, he joined Bell Communications Research, where he was a Distinguished Member of Staff and also Director of Solid-State Physics Research. In 1992, he joined the University of California at Los Angeles (UCLA), where he is Professor of electrical engineering. His work has covered a broad variety of topics, including nonlinear optics, laser-plasma interaction, infrared laser chemistry, photovoltaic energy conversion, strained-quantum-well lasers, and chemical modification of semiconductor surfaces. His current main interests are in optoelectronics, high-speed optical communications, high-efficiency light-emitting diodes and nanocavity lasers, photonic crystals at optical and microwave frequencies, quantum computing, and quantum communication.

Dr. Yablonovitch is a Fellow of the American Physical Society and the Optical Society of America (OSA), and is a member of Eta Kappa Nu. He chaired the 1979 Gordon Conference on Nonlinear Optics and Lasers. He has been the recipient of numerous honors and awards, including an Alfred P. Sloan Fellowship (1978-1979), the Adolf Lomb Medal of the Optical Society of America (1978), an R\&D 100 Award (1990), the W. Streifer Scientific Achievement Award presented by the IEEE Lasers and Electro-Optics Society (LEOS) (1993), and the R. W. Wood Prize of the Optical Society of America (1996).

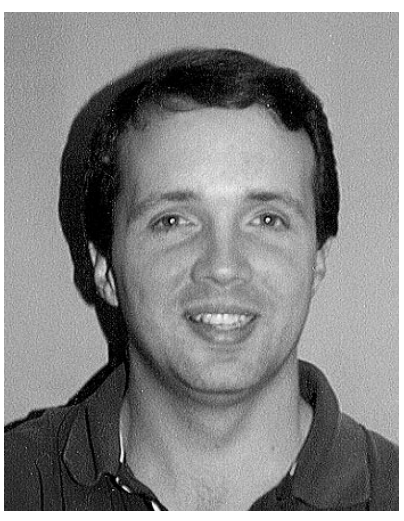

Henry O. Everitt was born in Huntsville, AL, in 1963. He received the B.S. degree in physics and mathematics and the Ph.D. degree in physics from Duke University, Durham, NC, in 1985 and 1990, resepctively.

$\mathrm{He}$ is currently a Program Manager for experimental condensed matter physics for the Army Research Office, Research Triangle Park, NC, and manages major extramural research programs in photonic-band engineering, nanoscience, and quantum information science. He is also an Adjunct Professor of physics at Duke University, Durham, NC, where he is currently leading two research efforts. The first is to develop a PC-based OPFIR laser while exploring the fundamentals of PC resonator design, and the second is an ultrafast optical characterization facility for the investigation of wide-bandgap semiconductor materials and devices.

John Aiden Higgins (M'62-SM'78-F'99) received the B.Eng. degree from University College Dublin, Dublin, Ireland, in 1958 and the M.S.E.E. and Ph.D. degrees in electrical engineering from Stanford University, Stanford, CA, in 1964 and 1971, respectively.

In 1971, he joined the Rockwell Science Center, Thousand Oaks, CA. Through 1981, he was a leading member of the team that pioneered the GaAs MESFET for microwave MMIC's. From 1981 to 1985, he developed multigigahertz GaAs charge coupled device (CCD) technologies for filter applications. From 1984 to 1997, he led the development of III-V compound semiconductor pHEMT and heterojunction bipolar transistor (HBT) MMIC technologies. In 1997, he became a Principal Scientist at Rockwell Science Center. His current activities include quasi-optic power amplifiers for millimeter waves, GaNbased high electron-mobility transistors (HEMT's), PXT's, microelectromechanical (MEMS) MMIC's, and CCD active filter technology. 